\title{
ACTIVE CONTROL OF QUARTER-CAR SUSPENSION SYSTEM USING LINEAR QUADRATIC REGULATOR
}

\author{
M.P. Nagarkar ${ }^{1}$, G.J. Vikhe ${ }^{2}$, K.R. Borole ${ }^{3}$ and V.M. Nandedkar ${ }^{4}$ \\ ${ }^{1}$ Ahmednagar Jilha Maratha Vidya Prasarak Samaj, Ahmednagar - 414001 (MS) INDIA. \\ Ph No.:+91-0241-2423887, +919860566496 \\ Email: maheshnagarkar@rediffmail.com \\ ${ }^{2}$ Principal, AV College of Engineering, Sangamner (MS) INDIA. \\ ${ }^{3}$ Department of Mechanical Engineering, Smt. Kashibai Navale College of Engineering \\ Pune - 411 041(MS) INDIA \\ ${ }^{4}$ Department of Production Engineering, S.G.G.S. Institute of Engineering and Technology \\ Nanded. (MS) INDIA
}

\begin{abstract}
The automobile is composed of many systems. One of these is the suspension system. The main functions of the automotive suspension system are to provide vehicle support, stability and directional control during handling manoeuvres and to provide effective isolation from road disturbances. The suspension system has to balance the tradeoff between ride comfort and handling performance. This paper analyses the passive suspension system and active suspension system using a Linear Quadratic Regulator (LQR) controller. A linear quarter-car model is used for the analysis and simulation. The performance of the LQR controller is compared with the passive suspension system. The simulation results show that the LQR controller improves vehicle ride comfort.
\end{abstract}

Keywords: active suspension system, LQR controller, quarter-car model.

\section{INTRODUCTION}

The automobile is a combination of a variety of complex systems. One such system is the suspension system. The suspension system has been widely applied to vehicles, from horsedrawn carriages with flexible leaf springs to modern automobiles with complex control algorithms. Passive suspension systems are a trade-off between ride comfort and performance. A car with a nice cushy ride usually wallows through the corners, whereas a car with high performance suspension, like F1 cars, will hang on tight through the corners but will make the passengers feel every little dip and bump in the road. The intent of the active suspension system is to replace the classical passive elements by a controlled system, an active suspension system, which can supply unlimited force to the system. The active suspension system dynamically responds to the changing road surface due to its ability to supply energy which is used to achieve the relative motion between the body and wheel. Toshimura et al. (2001) designed an active suspension system for a quarter-car model using the concept of sliding mode control. The active control is derived by the equivalent control and switching function while the sliding surface is obtained by using Linear Quadratic control (LQ control) theory. The experimental result indicates that the proposed active suspension system is more effective in the vibration isolation of the car body than the passive suspension system.

Sam et al. (2003) designed the proportional integral sliding mode control scheme. A quarter-car model is used in the study and the performance of the controller is compared to the existing passive suspension system. A simulation study proves the effectiveness and robustness of the control approach. Gürsel et al. (2006) utilized a PID controller for studying the performance of the active suspension system. Son and Isik (1996) described a fuzzy logic control method for an automotive active suspension system. The performance of the fuzzy logic 
controlled active suspension system is compared in several simulated scenarios. The performance of fuzzy control is much better than that of the passive suspension system for different road conditions and speeds. Neural Network Based Fuzzy Logic was developed by Wu et al. (2005) as an advanced self-learning optimal intelligent active suspension system. Artificial neural-based fuzzy modelling is applied to set up the neural-based fuzzy model based on the training data from the non-linear half-car suspension system dynamics. The development of self-learning optimal intelligent active suspension can not only absorb disturbance and shock, to adapt the model, the sensor and the actuator error, but also cope with the parameter uncertainty with minimum power consumption. The simulation results show that the designed active suspension system can improve the ride quality. In their study on LQG, Tamai and Sotelo (1995) presented preliminary results from a research about active suspension control regarding vehicle body flexibility using a LQG-LTR approach. Hrovat (1990) explores the connections between LQG-optimal one DOF and two DOF models. For the optimal two DOF systems, both ride and handling can be improved by reducing the unsprung mass. Here the maximum possible ride and handling improvements for two DOF systems are obtained in the limiting case of singular control with zero penalty on unsprung actuator force.

This paper presents a relatively simple active suspension control strategy - a Linear Quadratic Regulator (LQR). The LQR controller is used with a passive suspension system to improve the vehicle ride comfort. The model is subjected to disturbances like step, sine, white noise, bump, etc. The passive suspension system is used as a reference system. The performance of the LQR active suspension system is compared with the passive suspension system. The simulations were carried out in a MATLAB/Simulink environment.

\section{MATHEMATICAL MODELLING}

\section{Quarter-Car Model}

In order to analyse the behaviour of a dynamic system and to design a multivariable control for the same we need a mathematical model of the system. The model is constructed according to functional principles in view of the demand and is required to represent the kinematic and dynamic behaviour of the system in an equation. Many suspension models have been used by the researchers, namely the full car model, half-car model and quarter-car model. Kruczek and Stribrsky (2004) used a full car model by connecting four conventional quarter car suspension models. Tamai and Sotelo (1995) and Wu et al. (2005) used a four degrees of freedom half-car model to include heave and pitch motions in the front and rear wheels. The suspension system is modelled as linear viscous dampers and springs. McGee et al. (2005) used a non-linear quartercar model with non-linear forces like quadratic and cubic stiffness in the tyre and suspension and coulomb friction damping in suspension. A frequency domain technique is used to analyse the system. During the analysis, a linear quarter-car model is considered as it is simple to model, yet we can observe the basic elements of the suspension system, such as sprung and unsprung masses, sprung mass deflection, tyre deflection, and rattle/suspension space. Modelling of the suspension system is done in the vertical plane. Longitudinal or transverse deflections of the suspension components are considered negligible in comparison to vertical deflections. The complete vehicle mass is divided into two, i.e., the sprung mass and the unsprung mass. Springs and dampers are connected between the sprung and unsprung masses and unsprung mass and ground respectively, as shown in Figure 1.

\section{State Space Approach}

The second-degree differential equations of motion for the system can be written as follows:

$$
\begin{aligned}
& m_{1} \ddot{x}_{1}+\left(c_{1}+c_{2}\right) \dot{x}_{1}+\left(k_{1}+k_{2}\right) x_{1}-c_{2} \dot{x}_{2}-k_{2} x_{2}-c_{1} \dot{x}_{3}-k_{1} x_{3}=0 \\
& m_{21} \ddot{x}_{2}+c_{2} \dot{x}_{2}+k_{2} x_{2}-c_{2} \dot{x}_{1}-k_{2} x_{1}=0
\end{aligned}
$$


For the given system, 4 state variables are defined: two give the displacement of the two masses and the other two give the velocities of the respective masses.

Let

$$
\begin{aligned}
& x_{1}(t)=x_{2} ; \quad x_{2}(t)=x_{1} ; \quad x_{3}(t)=\dot{x}_{1}(t) ; x_{4}(t)=\dot{x}_{2}(t) \\
& \dot{x}_{3}(t)=\ddot{x}_{1}(t)=\ddot{x}_{2} ; \quad \dot{x}_{4}(t)=\ddot{x}_{2}(t)=\ddot{x}_{1}
\end{aligned}
$$

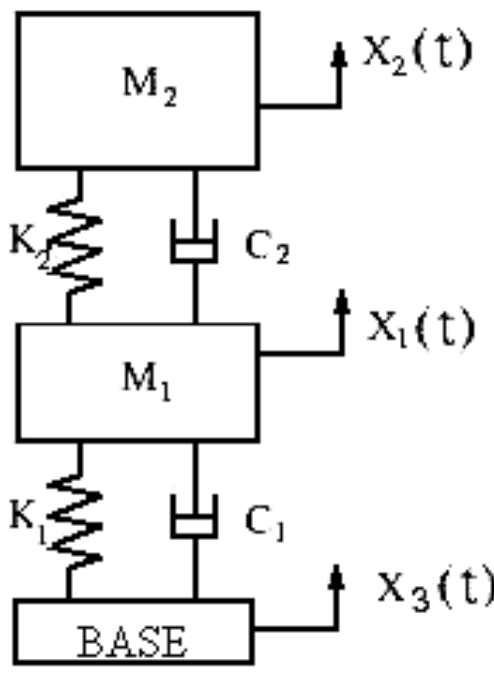

Figure 1. 2-DOF (Linear) system with base excitation - a quarter-car model.

Substituting Eq. (2) into Eq. (1), and finally re-arranging in the form of Eq. (3),

$$
\left.\begin{array}{l}
\dot{x}(t)=A x(t)+B u(t) \\
y(t)=C x(t)+D u(t)
\end{array}\right\}
$$

where $\dot{x}(t), x(t), y(t), A, B, C$ and $D$ are the matrices of various orders.

Matrix $A$ is called the state matrix, $B$ is the input matrix, $C$ is the output matrix and $D$ is the direct transmission matrix.

$$
\begin{gathered}
A=\left[\begin{array}{cccc}
0 & 0 & 1 & 0 \\
0 & 0 & 0 & 1 \\
-k_{2} / m_{2} & k_{2} / m_{2} & -c_{2} / m_{2} & c_{2} / m_{2} \\
k_{2} / m_{1} & -\left(k_{1}+k_{2}\right) / m_{1} & c_{2} / m_{1} & -\left(c_{1}+c_{2}\right) / m_{1}
\end{array}\right] \\
B=\left[\begin{array}{c}
0 \\
0 \\
0 \\
k_{1} / m_{1}
\end{array}\right] ; \quad C=\left[\begin{array}{cccc}
1 & 0 & 0 & 0 \\
0 & 1 & 0 & 0 \\
0 & 0 & 1 & 0 \\
0 & 1 & 0 & 1
\end{array}\right] ; \quad D=\left[\begin{array}{l}
0 \\
0 \\
0 \\
0
\end{array}\right]
\end{gathered}
$$




\section{LQR CONTROLLER}

For the design of a stable control system based on quadratic performance indexes, let us consider that the system is expressed as Eq. (4):

$$
\dot{x}=A x+B u
$$

where $x=$ state vector (n-vector); $u=$ control vector(r-vector); $A=n \times n$ constant matrix

$B=n \times r$ constant matrix.

In designing control systems, one is often interested in choosing the control vector $u(t)$ such that a given performance index is minimized. The quadratic performance index is expressed as Eq. (5).

$$
J_{L Q R}=\int L(x, u) d t
$$

where $L(x, u)$ is a quadratic function or Hermitian function of $x$ and $u$, leading to a linear control law.

$$
u(t)=-L_{L Q R} x
$$

where $K_{L Q R}$ is a $L Q R$ gain matrix.

In linear quadratic regulator control, the quadratic performance index is expressed as Eq. (7).

$$
J_{L Q R}=\int\left(x^{T} Q x+u^{T} R u\right) d t
$$

where the matrix $Q$ is a positive-definite (or positive-semi definite) Hermitian or real symmetric matrix, $R$ is a positive definite Hermitian or real symmetric matrix. The first term on the right-hand side of the equation accounts for the error between the initial and final state, and the second term accounts for the expenditure of the energy of the control signal. The matrices $Q$ and $R$ determine the relative importance of the error and expenditure of the performance index. The control vector $u(t)$ is considered to be unconstrained.

The control law given by Eq. (6) is the optimal control law. Therefore, if the unknown elements of the matrix $K$ are determined so as to minimize the performance index, then $u(t)=-K_{L Q R x}$ is optimal for any initial state $x(0)$. The block diagram of the optimal configuration is shown in Figure 2.

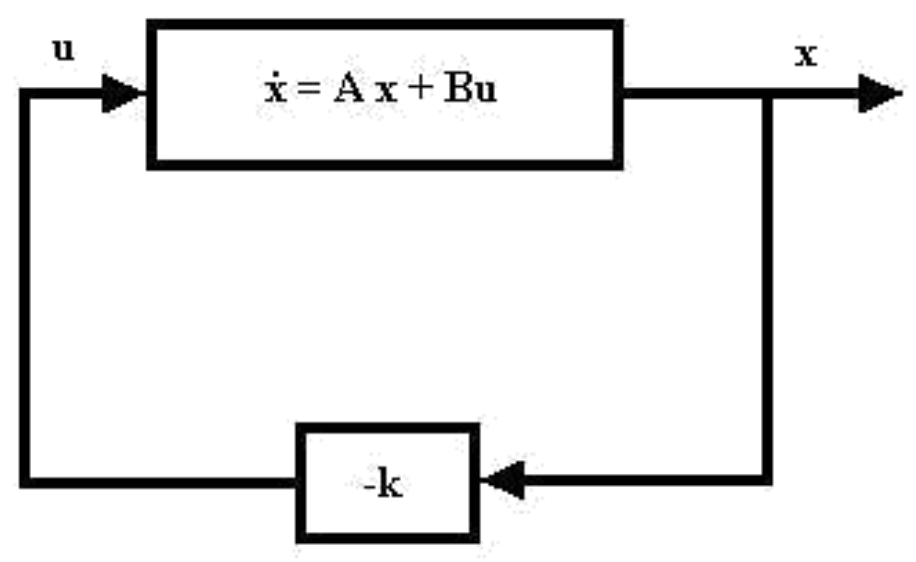

Figure 2. Block diagram of LQR control scheme. 


\section{LQR CONTROLLER DESIGN}

In linear quadratic control, the control input is

$$
u(t)=-L_{L Q R} x
$$

The system equation is

$$
\dot{x}=A x+B u
$$

Then, substituting Eq. (6) into Eq. (4),

$$
\dot{x}=A x-B K_{L Q R} x=\left(A-B K_{L Q R}\right) x
$$

We assume that the matrix $\left(A-B K_{L Q R}\right)$ is stable or that the eigenvalues of $A-B K$ have negative real parts. Substituting Eq. (6) into Eq. (7) we have

$$
J_{L Q R}=\int_{0}^{\infty}\left(x^{T} Q x+x^{T} K_{L Q R}^{T} R K_{L Q R} x\right) d t=\int_{0}^{\infty}\left(x^{T}\left(Q+K_{L Q R}^{T} R K_{L Q R}\right) x\right) d t
$$

To solve the above parameter optimizing problem, let us set

$$
x^{T}\left(Q+K_{L Q R}^{T} R K_{L Q R}\right) x=-\frac{d}{d t}\left(x^{T} P x\right)
$$

where $P$ is a positive definite matrix.

Now Eq. (10) can be rewritten as

$$
\begin{aligned}
& x^{T}\left(Q+K_{L Q R}^{T} R K_{L Q R}\right) x=-\dot{x}^{T} P x-x^{T} P \dot{x} \\
& =-x^{T}\left(A-B K_{L Q R}\right)^{T} P x+x^{T} P\left(A-B K_{L Q R}\right) x \\
& =-x^{T}\left[\left(A-B K_{L Q R}\right)^{T} P-x^{T} P\left(A-B K_{L Q R}\right)\right] x
\end{aligned}
$$

Comparing the RHS and LHS of the above equation and noting that the equation must hold true for any $x(t)$, we require that:

$$
\left(A-B K_{L Q R}\right)^{T} P-x^{T} P\left(A-B K_{L Q R}\right)=-\left(Q+K_{L Q R}^{T} R K_{L Q R}\right)
$$

The $A-B K_{L Q R}$ is a stable matrix, and there exists a positive definite matrix $P$ that satisfies Eq. (12). The performance index $J_{L Q R}$ can be evaluated as:

$$
\begin{aligned}
& J_{L Q R}=\int_{0}^{\infty}\left(x^{T}\left(Q+K_{L Q R}^{T} R K_{L Q R}\right) x\right) d t=\int_{0}^{\infty}\left[-\frac{d}{d t}\left(x^{T} P x\right)\right] d x=\left[-x^{T} P x\right]_{0}^{\infty} \\
& =-x^{T}(\infty) P x(\infty)+x^{T}(0) P x(0)
\end{aligned}
$$

Since all eigenvalues of $(A-B K)$ are assumed to have negative real parts, we have $x(\infty) \rightarrow$ 0. So, we have obtained Eq. (14):

$$
J_{L Q R}=x^{T}(0) P x(0)
$$


From Eq. (14), we can observe that the quadratic cost function $J_{L Q R}$ depends only on the values of $P$ as $x(0)$ is constant. Hence, to minimize the value of $J$, for given $x(0)$ with respect to $K$, we set

$$
\frac{\partial P}{\partial K}=0
$$

Therefore, differentiating Eq. (12) with respect to $K$ and substituting $\partial \mathrm{P} / \partial \mathrm{K}=0$, we obtain the value of $K$, which minimizes $J_{L Q R}$ as Eq. (16):

$$
K_{L Q R}=R^{-1} B^{T} P
$$

\section{RESULTS AND DISCUSSION}

The simulation is carried out in a Matlab/SIMULINK ${ }^{\circledR}$ environment. For the simulation, it is assumed that the vehicle hits a step, a white noise, a bump, a triangular bump, city road conditions and highway road conditions. The input data to the Simulink model are as follows:

$$
\begin{array}{lll}
m_{l}=40 \mathrm{~kg}, & k_{l}=124660 \mathrm{~N} / \mathrm{m}, & c_{l}=414 \mathrm{Ns} / \mathrm{m} \\
m_{2}=243 \mathrm{~kg}, & k_{2}=14671 \mathrm{~N} / \mathrm{m}, & c_{2}=370 \mathrm{Ns} / \mathrm{m} .
\end{array}
$$

The result shows the displacement, acceleration of sprung mass and the rattle space or the suspension space deflections, i.e., $x_{2}(t)-x_{1}(t)$.

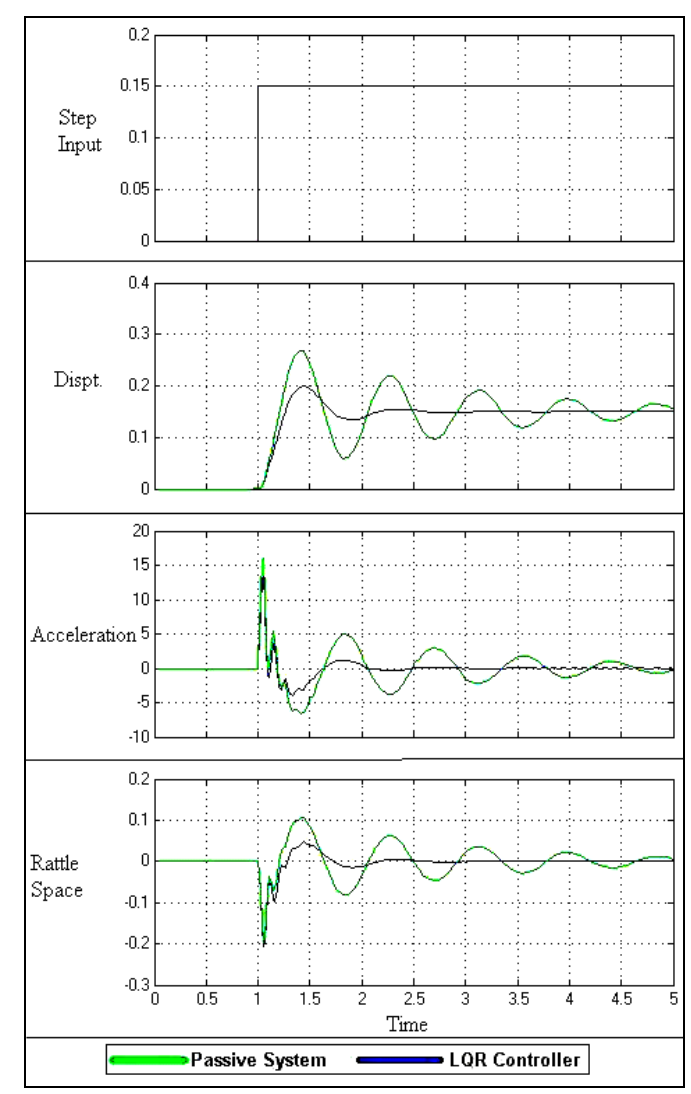

(a) Step input

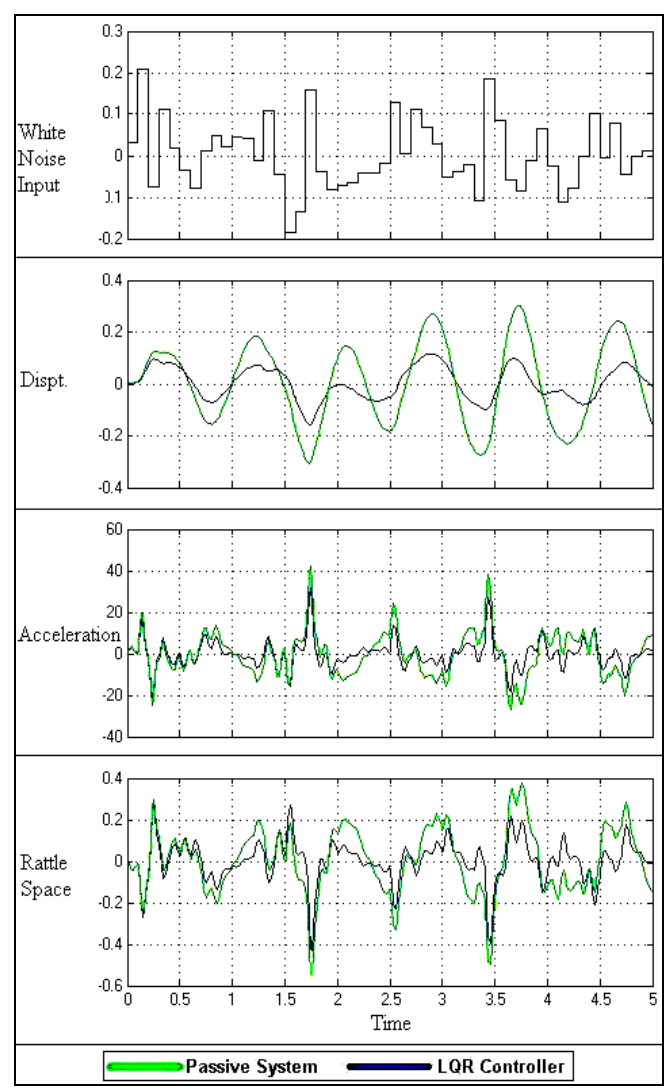

(b) White noise

Figure 3. Responses of passive system and LQR controller for (a) step input, (b) white noise. 


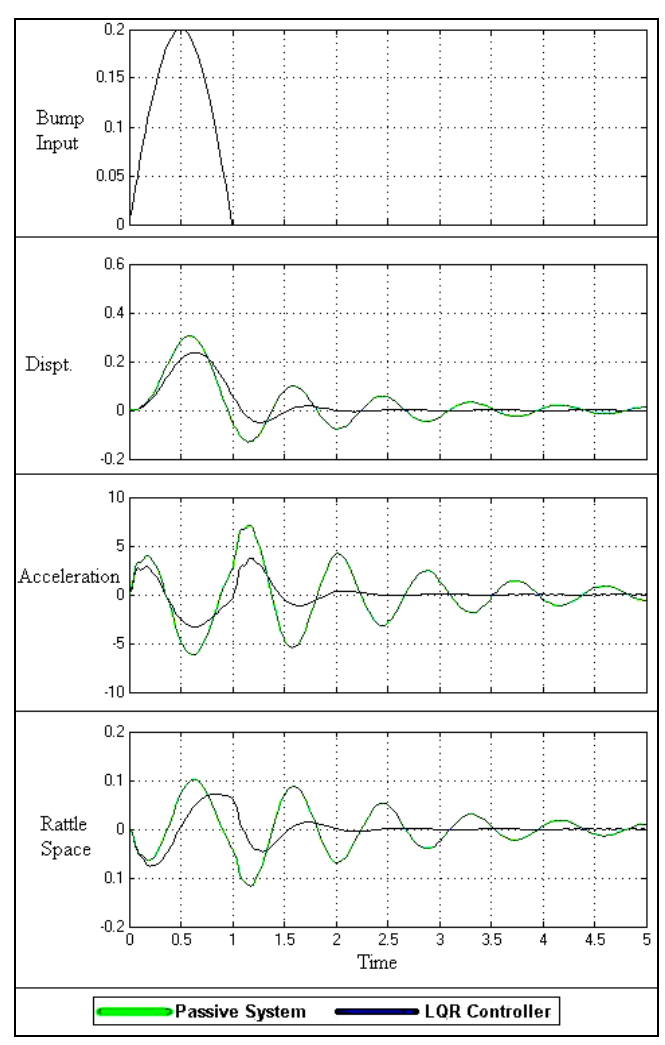

(a) Bump input

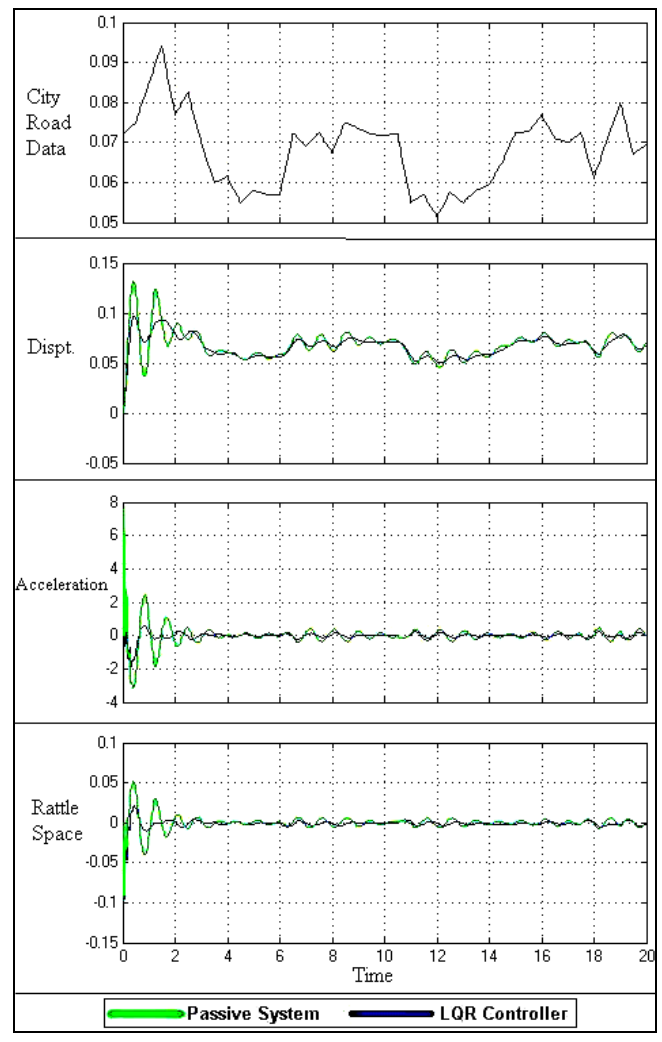

(c) City road data input

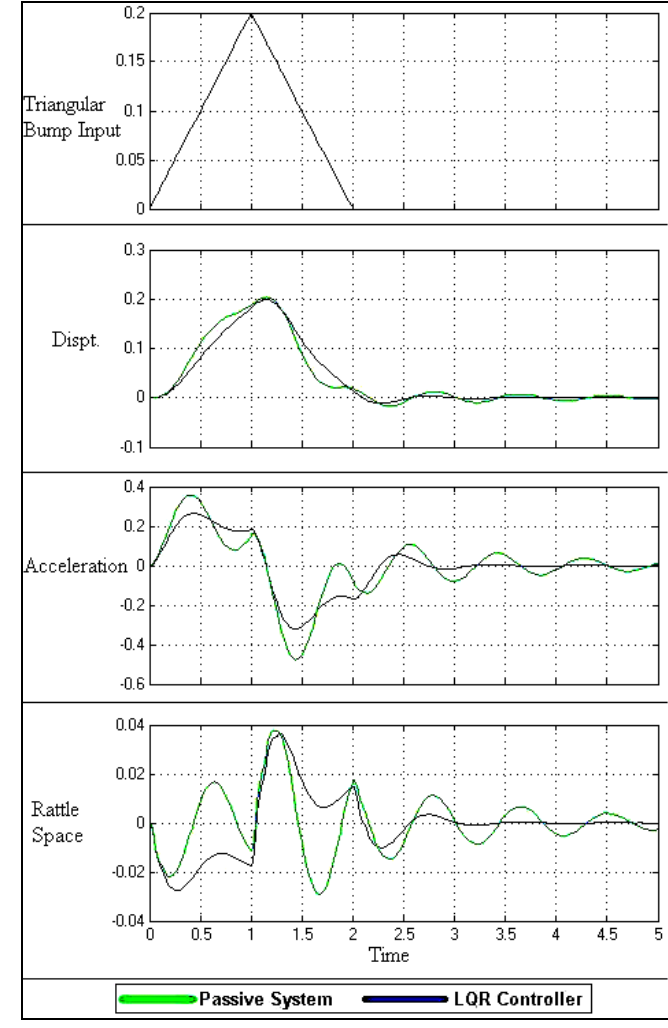

(b) Triangular bump input

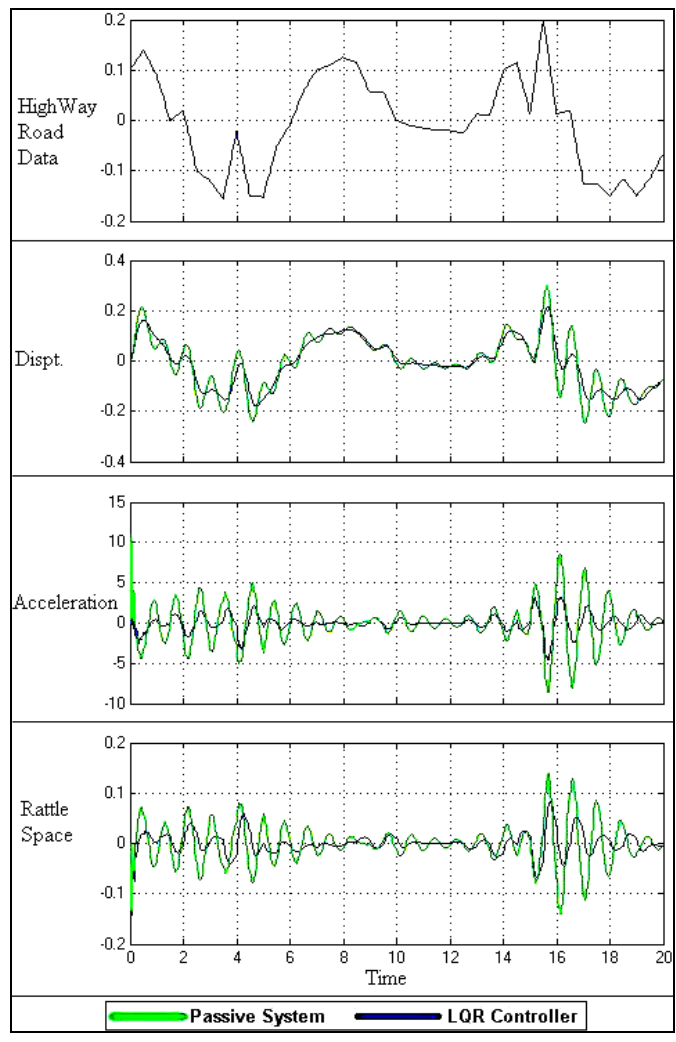

(d) Highway road data input

Figure 4. Responses for various conditions. 
Figures 3 and 4 show that the active suspension system utilizing the LQR controller performed better than the conventional passive suspension system. These figures illustrate clearly how the active suspension system using a LQR controller can absorb the vehicle vibrations more effectively than the conventional passive suspension system. The body acceleration in the LQR system is reduced significantly, which guarantees better ride comfort. Moreover, the wheel suspension deflection is also smaller than with the passive system.

\section{CONCLUSIONS}

The main aim of this paper is to demonstrate the active control strategy - the LQR controller against a passive suspension system. We have developed a LQR controller to enhance the ride comfort of passengers. A passive suspension system without any controller and an active suspension system with the LQR controller were modelled and simulated using a Matlab/Simulink environment. The simulation result shows that the designed active suspension system can improve the ride quality by minimizing the displacements and acceleration more than the passive suspension system. This means that the active suspension system using the LQR controller provides better ride comfort. In the active suspension systems using the LQR controller the rattle space or suspension space requirements remain smaller than with the conventional passive suspension system. Also, the LQR controller is tested against various test signals like step, sinusoidal, white noise, bump, triangular bump city road data and highway road data. Thus, the LQR controller demonstrates its flexibility by showing its ability to handle various kinds of road conditions.

\section{REFERENCES}

Anderson, B. and Moore, J. 1990 Optimal control - linear quadratic methods. NJ: Prentice Hall.

Balachandran, B. and Magrab, E.B. 2006. Vibrations. First Reprint. Singapore: Thomson Brooke/Cole.

Elmadany, M.M. 1989. Design of an active suspension for a heavy duty truck using optimal control theory. Computers and Structures, 31(3): 385-393.

Filler, C.R., Elliott, S.J. and Nelson, P.A. 1996. Active control of vibrations. London: Academic Press.

Gürsel, N. Altas, I.H. and Gümüsel, L. 2006. Fuzzy control of a bus suspension system. Proceedings of 5th International Symposium on Intelligent Manufacturing Systems, pp. 1170-1177.

Hrovat, D. 1990. Optimal active suspension structures for quarter-car vehicle models. Automatica, 26(5): 845-860.

Hrovat, D. 1997. Survey of advanced suspension developments and related optimal control applications. Automatica, 33(10): 1781-1787.

Kruczek, A. and Stribrsky, A. 2004. A full-car model for active suspension - some practical aspects. ICM Proceedings of the IEEE International Conference on Mechatronics, pp. 41-45.

McGee, C.G., Haroon, M. and Adams, D.E. 2005. A frequency domain technique for characterizing nonlinearities in a tire-vehicle suspension system. ASME Journal of Vibration and Acoustics, 127: 61-76.

Ogata, K. 2002. Modern Control Engineering. Fourth Edition. India: Prentice Hall.

Sam, Y.M., Osman, J.H.S. and Ghani, M.R.A. .2003. Active Suspension Control: Performance Comparison using Proportional Integral Sliding Mode and Linear Quadratic Regulator Methods. Proceedings of IEEE Conference on Control Applications, Istanbul, Turkey, pp. 274-278.

Son, S.I. and Isik, C. 1996. Application of Fuzzy Logic Control to an Automotive Active Suspension System. Proceedings of the Fifth IEEE International Conference on Fuzzy Systems, pp. 548-553. 
Tamai, E.H. and Sotelo, J. 1995. LQG - control of active suspension considering vehicle body flexibility. Proceedings of 4th IEEE Conference on Control Applications, pp. 143-147.

Tang, C. and Zhang, T. 2005. The research on control algorithms of vehicle active suspension system. Proceedings of IEEE International Conference on Vehicular Electronics, pp. 320-325.

Toshimura, T., Kume, A., Kuromoto, M. and Hino, J. 2001. Active suspension system of a quarter car using the concept of sliding mode control. Journal of Sound and Vibration, 239: 187-199.

Wu, S.J., Wu, C.T. and Lee, T.T. 2005. Neural-network-based fuzzy control design for half-car active suspension systems. Proceedings of IEEE Intelligent Vehicles Symposium, pp. 376-381. 